and for the detection of causal associations among these complex conditions.

At the same time the study underlines the need to extend and refine available techniques of measurement. Even with carefully defined criteria of illness the wide variation between practitioners' prevalence rates points to two potent groups of factors. The first of these relates to the ecological differences between practices and can be offset by paying regard to the size and representativeness of the sample of participating doctors. The second relates to the doctor, his attitudes, and his interactions with his patients; for psychiatric illness, as we have shown, they constitute a major source of variation.

\section{Summary}

This paper reports on a general practice survey of psychiatric morbidity carried out in the Greater London area. The sickness experience of nearly 15,000 patients on the lists of 80 practitioners was studied over a period of one year. It was shown that minor psychiatric disorders, classified according to a predetermined schema, were diagnosed in $14 \%$ of the population at risk. Neurotic illness was most prevalent among middle-aged females and proved to be an important cause of chronic illness. The reasons for interpractice variation in recorded morbidity are discussed.

We are grateful to the following general practitioners, whose cooperation made this study possible.

D. Adderley, G. J. Arendt, J. B. Armstrong, June Armstrong.
D. Berry, D. Billig, A. Blofeld, E. Bronson, H. Brostoff.
R. Clipstein, J. H. Clough, J. Cohen, N. Cohen.

J. Dillane.

G. M. Edmonds, H. H. A. Elder.

G. M. Edmonds, H. H. A. Elder.

Fletcher, K. B. Foster, P. Frazer, P. Freeman, J. Fry, L. Fry.

R. Grahame, D. Grant.

S. P. Halpin, J. C. Hardy, H. Hart, J. Hewetson, E. H. Hiley, P. Hopkins, J. Horder, G. Horton, K. . Huntington.

H. Jeffs, C. Josephs.

M. A. Kalina, C. O. Kennedy, D. L. Kerr, C. D. Korn, R. Korn.

S. Lee, B. A. Lees, H. N. Levitt, F. Lucas.

W. T. MacDonald, J. McEwan, T. Madden, D. H. Mellins, S. Mellins,

P. Morris, W. B. Mumford.

R. H. V. Ollendorff.

L. T. Page, D. Perkins, J. Powell-Evans.

J. N. Rea, F. Ross, E. M. Rosser, S. S. Rowell, A. Ryle.

I. J. Sachs, K. Sanders, J. Sayers, K. Scott, M. Scott, J. E. Scriven, E. Seifert, M. Smith, H. Snowden, A. Spier, R. Stowe. M. Thorpe, E. D. Tod.

J. Vance. Wall, T. H. Warburton, J. W. Wigg, R. Wigoder.

REFERENCES

Brown, A. C., and Fry, J. (1962). 7. psychosom. Res., 6, 185.

Cooper, B. (1964). De Medicina tuenda, 1, 43.

Brown, A. C., and Kalton, G. (1962). 尹. Coll. gen. Practit., 5, 590. Crombie, D. L. (1963). Lancet, 1, 1205.

Culpan, R. H., Davies, B. M., and Oppenheim, A. N. (1960). Brit. med. F., 1, 855 .

Eimeri, T. S. (1960). 7. Coll. gen. Practit., 3, 246.

County Report, London. H.M.S.O., London.

Kessel, W.. I. N. (1960). Brit. F. prev. soc. Med., 14, 16.

Logan, W. P. D., and Cushion, A. A. (1958). Studies on Medical and Population Subjects, No. 14. H.M.S.O., London.

Shepherd, M., Fisher, M., Stein, L., and Kessel, W. I. N. (1959). Proc. roy. Soc. Med., 52, 269.

W.H.O. (1948). International Statistical Classification of Diseases, Injuries, and Causes of Death. Geneva.

\title{
Haemodynamic Effects of Carotid Artery Stenosis
}

\author{
J. G. BRICE,* M.B., F.R.C.S. ; D. J. DOWSETT, $\dagger$ B.SC. ; R. D. LOWE, $\ddagger$ M.D., M.R.C.P.
}

Brit. med. F., 1964, 2, 1363-1366

Though atherosclerosis of carotid and vertebral arteries is common its significance is debatable. Hutchinson and Yates $(1957,1961)$ found a high incidence in a post-mortem series of patients who had had a cerebrovascular accident, but they did not study any control subjects; they suggested that extracranial arterial disease was a common cause of cerebral infarction through its direct effect on cerebral blood flow. Schwartz and Mitchell (1961) showed that atherosclerosis of these arteries, as with atherosclerosis elsewhere, correlated well with age, that it was common even in patients without cerebrovascular accidents, and that a correlation between cerebral infarction and extracranial atherosclerosis could be due largely to the fact that both were correlated with age.

The occlusive lesions described by these authors varied from small plaques scarcely narrowing the arterial lumen to complete occlusion of one or more of the extracranial arteries. The incomplete lesions were classified, according to the percentage reduction of the normal lumen, into mild or moderate (less than $50 \%$ reduction) or severe (more than $50 \%$ reduction); no evidence is available to assess the possible effects of such stenosis on cerebral blood flow.

Alteration of calibre may be of importance either directly, by its effect on cerebrovascular resistance, or indirectly, producing turbulent conditions of flow which might predispose to local platelet aggregation. In this paper we are concerned with the effect of narrowing of carotid arteries on their hydraulic resistance only, and report experiments designed to determine the approximate dimensions and hydraulic resistance of the minimum narrowing which has a detectable effect on blood flow through the artery. We have measured pressure gradient and flow in the human carotid artery and defined a degree of constriction which has just detectable haemodynamic effects ; in order to assess the influence of shape and length we have then reproduced this constriction in vitro in a perfusion circuit and matched its hydraulic resistance with that of artificial resistances. Some of these results have already been briefly reported (Brice, Dowsett, and Lowe, 1964).

\section{Methods}

Apparatus.-Carotid artery flow was measured by a gated sine-wave electromagnetic flowmeter (Medicon FM6) using an external probe. Pressure was measured at two points about $5 \mathrm{~cm}$. apart, using 22-gauge needles inserted into the carotid artery and connected by $80 \mathrm{~cm}$. of nylon tube (No. 2 nylon,

\footnotetext{
* Research Assistant, Neurosurgical Department and Medical Unit, St. George's Hospital, London.

† Research Assistant, Neurosurgical Department and Medical Unit, St. George's Hospital, London. Wellcome Senior Research Fellow, Neurosurgical Department and
Medical Unit, St. George's Hospital, London.
} 
Portland Plastics) to Statham P23Db pressure transducers. The signals were amplified by transistor amplifiers and recorded on a Cambridge four-channel galvanometer recorder. Both mean flow and mean pressure were calculated as the diastolic figure plus one-third of the pulse amplitude. At the amplification used the smallest changes that could be detected were about 3 $\mathrm{mm}$. $\mathrm{Hg}$ in the pressure signal and $7 \mathrm{ml} . /$ minute in the flow signal.

The artery was constricted by one of two devices. One was a specially made micrometer clamp with parallel blades with which a pre-set gap could be selected and the constriction apolied suddenly; the blades were $3 \mathrm{~mm}$. wide and $14 \mathrm{~mm}$. long. The other was a standard Preston carotid clamp with blades $10.5 \mathrm{~mm}$. square ; the artery was constricted by progressive tightening and the gap had to be calculated from the pitch of the screw.

Subjects.-Studies were attempted in 13 subjects and were successful in eight using simultaneous pressure and flow recordings ; in a further eight subjects only pressure recordings were made. All subjects were patients with intracranial berry aneurysms, on or near the circle of Willis, whose common carotid arteries were to be ligated. The normal operative procedure was to open the neck under local anaesthesia supplemented by methohexitone, and then to allow the patient to recover consciousness while the common carotid artery was clamped for a trial period of 20 minutes. The additional operative procedure required by our investigation was to use a larger incision and to dissect out the carotid bifurcation; all experiments were carried out with the patient conscious, to ensure that carotid blood flow was not reduced by anaesthesia.

In vivo Procedures. - The diastolic diameter of the common carotid artery was measured with the micrometer clamp and a flow-probe applied whose diameter was about $1 \mathrm{~mm}$. less. The external carotid artery was occluded by a bulldog clamp (so that only internal carotid flow was recorded), and pressure recording needles were inserted. The artery was constricted between the needles, using constrictions of increasing severity until flow was zero and pressure gradient maximal (the point of "zero flow" occurred with a constriction about $0.1 \mathrm{~mm}$. wider than the point of maximal pressure gradient); the gap between the blades of the clamp was now twice the effective wall-thickness, and from this figure and from the measured width of the artery in the clamp the cross-sectional area of the slit-like lumen could be calculated for lesser degrees of constriction.

In vitro Procedures.-A constant-flow perfusion circuit was used with a $25 \%$ solution of sucrose in water at $20^{\circ} \mathrm{C}$., flowing at $160 \mathrm{ml} . / \mathrm{min}$. This fluid has a viscosity of 0.0245 poise, which is in the same range as the viscosity of blood. An excised human carotid artery was connected into the circuit at a pressure of approximately $100 \mathrm{~mm}$. $\mathrm{Hg}$ and its resistance measured. Artificial resistances of different shapes and length were introduced into a rubber tube of suitable bore and their resistance was measured under the same conditions. To verify that at the point of maximal pressure gradient obtained in vivo the artery was completely occluded, an excised artery was constricted in the same way and perfused with saline at a pressure of $100 \mathrm{~mm}$. $\mathrm{Hg}$; flow was recorded by electromagnetic flowmeter at the same amplification as was used in vivo. It was found that the flowmeter recorded zero flow when the lumen of the constriction was about $0.05 \mathrm{~mm}$. across, although saline was still flowing at a few $\mathrm{ml} . / \mathrm{min}$. This gap corresponds approximately with the observed difference in vivo between the degrees of constriction producing "zero flow" and maximal pressure gradient. To verify the actual cross-sectional area of the constricted segment five excised arteries were perfused at $100 \mathrm{~mm} . \mathrm{Hg}$ and subjected to exactly the same procedures in vitro, calculating lumen size in the same way. Casts of the constricted arteries were then made using an epoxy resin; the casts were cut across at the thinnest point, and the crosssectional area was measured with a microscope and graticule scale.

\section{In Vivo Results}

The usual difficulties were met in using the electromagnetic $\stackrel{\text { एक }}{\frac{\text { }}{3}}$ flowmeter-difficulty in obtaining a constant zero-flow baseline $\mathbb{0}$ and movement artifacts. The very act of occlusion sometimes ${ }_{C}$ disturbed the position of the flow-probe relative to the artery and caused a shift of baseline; the pulsatile movement of thes? artery longitudinally inside the probe caused transient signalso unless the probe was free to move with the artery and did not nudge against surrounding tissues. With these difficulties the $\frac{\bar{s}}{\frac{1}{2}}$ absolute values of flow are open to question and results are $\mathbb{\complement}$ therefore expressed as percentage changes in the resting flow ;
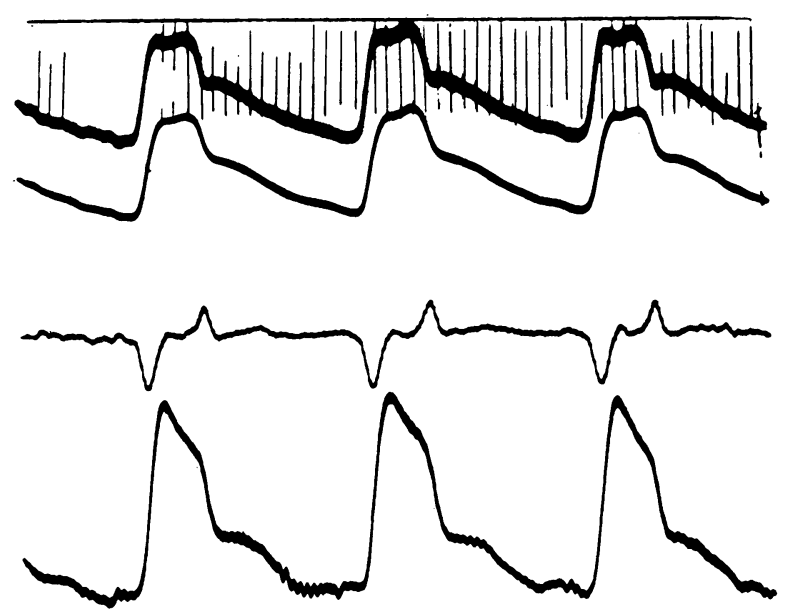
FIG. 1.-A sample experimental record showing, from above downwards, verted), and carotid artery flow. The time-marker intervals represent 0.04 second.

it was usually necessary to damp the signal to reduce transient signals due to movement, and results are therefore based on the mean flow. Fig. 1 illustrates the best record of undamped $\stackrel{\mathbb{\Phi}}{\varrho}$ pulsatile flow and pressure.

Results in a typical subject are illustrated in Fig. 2. With $\frac{0}{3}$ progressive constriction of the artery there was at first no? change of pulse pressure, of mean pressure, or of flow ; further: narrowing abruptly produces a steep change of distal pulse pressure, mean gradient, and flow. Results in a further seven subjects were very similar; using blades of $3 \mathrm{~mm}$. width for:constriction, the mean cross-sectional area of the constriction 3 . which first produced detectable effects on pressure and flow was 4.07 sq. mm. (standard deviation 0.693 ); the mean crosssectional area of the constriction which just failed to have $\mathrm{O}$ detectable effects was 6.23 sq. mm. (standard deviation 0.96). Between these two figures a "critical" cross-sectional area cano be distinguished; we have arbitrarily defined this as the area of that constriction which would produce a $5 \%$ fall of distal mean pressure and a $10 \%$ fall of mean flow. This "critical "N area averaged 4.5 sq. $\mathrm{mm}$. (standard deviation 0.73 ) and bore noN

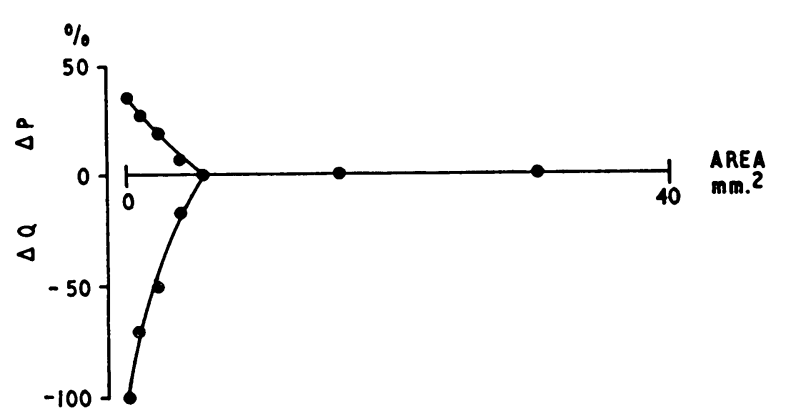

Fig. 2.-In vivo results from one subject. The abscissa shows the cross sectional area at the site of constriction ranging from the normal area of the artery (right-hand point) downwards. The ordinate shows the percentage changes in mean pressure gradient $(\Delta \mathrm{P})$ and mean flow $(\Delta \mathrm{Q})$ centage changes in mean pressure gradient 
relation to the normal cross-sectional area of the artery (Fig. 3). In a further eight subjects, using pressure recordings only, the arrery was constricted by a Preston clamp which produced a constriction $10.5 \mathrm{~mm}$. long. The "critical" area for these constrictions was 4.6 sq. $\mathrm{mm}$. (standard deviation 0.65 ).

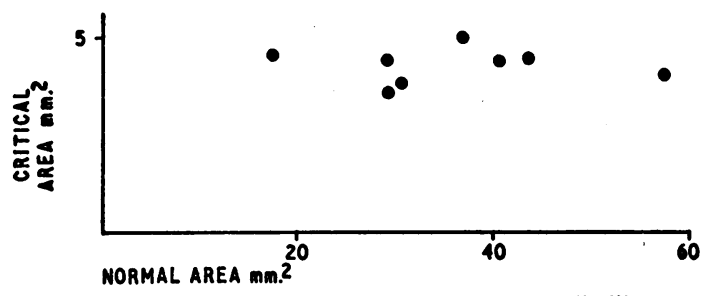

FIG. 3.-In vivo results from eight subjects, using a slit-like constriction $3 \mathrm{~mm}$. long. The "critical" area (see text) is plotted against the normal cross-sectional area of the unconstricted artery for each subject.

\section{In Vitro Results}

The shape of the constriction produced by the micrometer clamp is illustrated in Fig. 4 ; the region of greatest narrowing

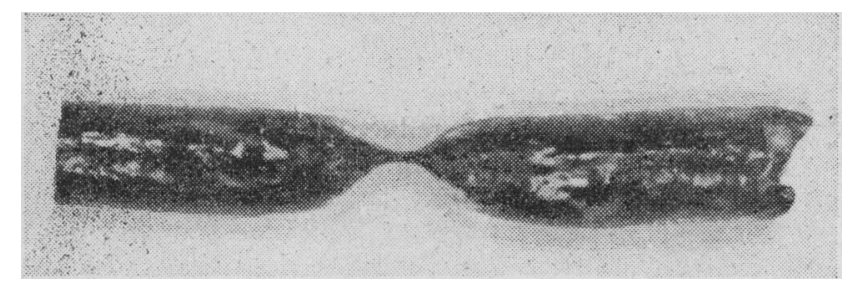

FIG. 4.-An in vitro cast of a slit-like constriction in a carotid artery. The segment of greatest narrowing is $3 \mathrm{~mm}$. long and has a crossstriction without detectable effect on pressure gradient or flow.

was approximately $3 \mathrm{~mm}$. long, with a smooth inlet and outflow tract. The cross-sectional area measured in five casts did not differ significantly from the area calculated for the same constriction in perfusion experiments.

Fig. 5 illustrates the influence of cross-sectional area on resistance for resistances of different lengths and shapes. Curve $\mathrm{C}$ was obtained in an artery with slit-like constrictions $3 \mathrm{~mm}$. long, having the shape of slit illustration in Fig. 4 ; the hydraulic resistance of a "critical" constriction 4-5 sq. mm. in area is $6.25 \mathrm{~mm}$. $\mathrm{Hg} / 100 \mathrm{ml} . / \mathrm{min}$. Curve A shows the results obtained in a rubber tube using artificial resistances

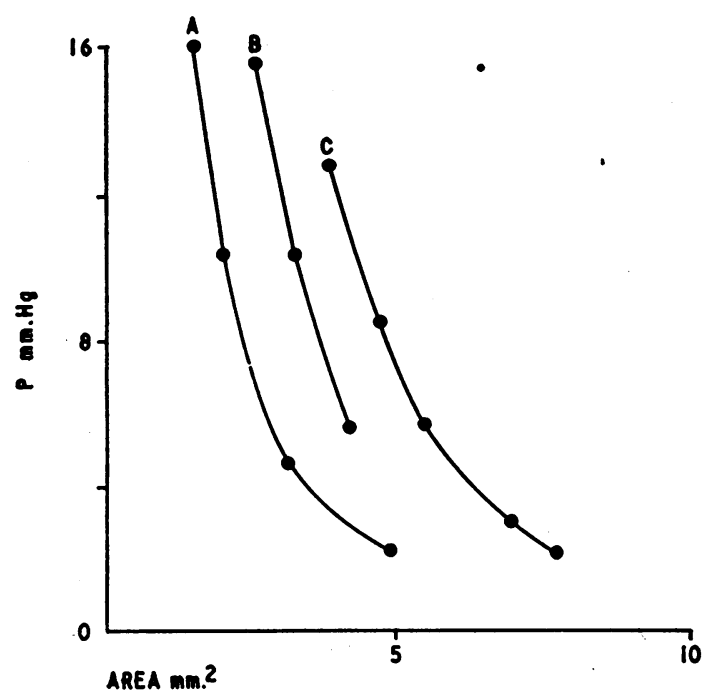

Fig. 5.-In vitro results of perfusion experiments using $25 \%$ sucrose flowing at $160 \mathrm{ml}$./min. through a constriction. The cross-sectional area flowing at $160 \mathrm{ml}$. min. through a constriction. The cross-sectional area
of the lumen is plotted against the pressure gradient ; $\mathbf{A}$, round constriction $3 \mathrm{~mm}$. long; $\mathrm{B}$, round constriction $30 \mathrm{~mm}$. long; C, slit-like constriction $3 \mathrm{~mm}$. long. The "critical" resistance at this flow rate has a pressure gradient of $10 \mathrm{~mm}$. $\mathrm{Hg}$. with circular cross-section and inlet and outflow tracts at right angles to the wall ; the resistance of a "critical " slit-like constriction is duplicated by a round constriction $2.1 \mathrm{sq} . \mathrm{mm}$. in area. Curve $B$ shows results with a round constriction with a length of $30 \mathrm{~mm}$. instead of $3 \mathrm{~mm}$. ; the critical resistance is duplicated at an area of 3.3 sq. $\mathrm{mm}$.

The effect of length of the constriction upon resistance is shown in more detail in Fig. 6, using a round constriction of area $2.6 \mathrm{sq}$. $\mathrm{mm}$. The total resistance is the sum of two factors: one is determined by the geometry of the inflow and outflow tracts and is not length-dependent; the other is due to the narrowed segment itself and is dependent upon the length; the intercept of the line with the $y$ axis gives the resistance of a diaphragm constriction of no effective length. From this figure it can be seen that the change of resistance with length is relatively small, and it is clear why experiments in vivo failed to distinguish between constrictions $3 \mathrm{~mm}$. and $10.5 \mathrm{~mm}$. long.

Fig. 7 illustrates an experiment which demonstrates that two constrictions in series do not necessarily have a total resistance equal to the sum of their separate resistances ; when two resistances are contiguous, each of $1 \mathrm{~cm}$. length, their resistance is the same as that of a resistance $2 \mathrm{~cm}$. long (see Fig. 6) ; as their separation increases their total resistance increases until at a separation of about $3 \mathrm{~cm}$. their total resistance is equal to the sum of their separate resistances. From Fig. 5 it can be calculated that the "critical " resistance would be duplicated

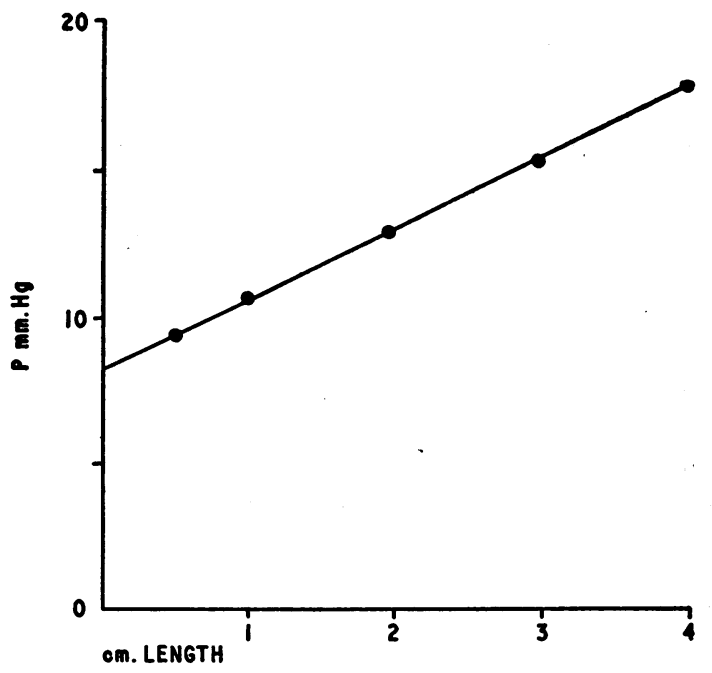

FIG. 6.-Influence of length of constriction upon pressure gradient at constant flow, using a round constriction of 2.6 sq. $\mathrm{mm}$. cross-sectional area (experiment as in Fig 5 ).

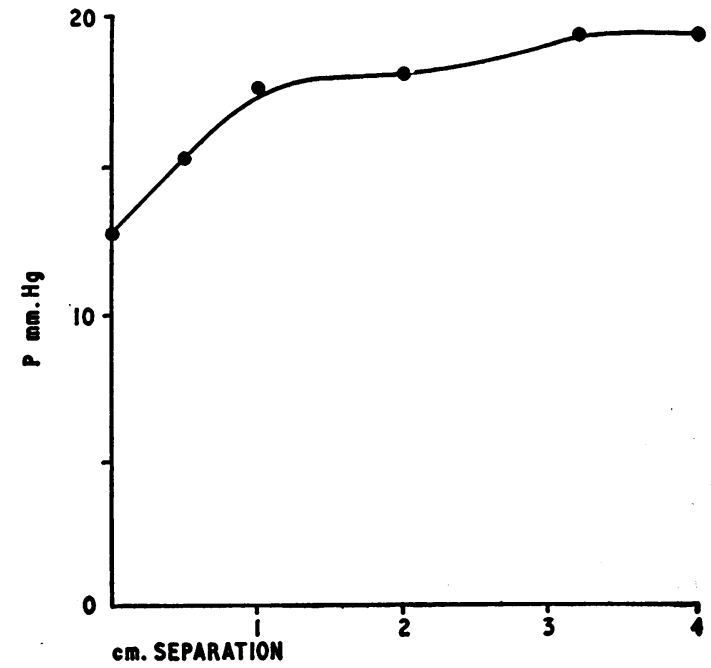

FIG. 7.-Effect of two separate constrictions in series, each $100 \mathrm{~mm}$. long and 2.6 sa. $\mathrm{mm}$. in bore. The pressure gradient across both constrictions is plotted against the distance separating them (experiment as in Fig. 6). 
by two round constrictions, each having a bore of 3.2 sq. $\mathrm{mm}$. and a length of $3 \mathrm{~mm}$.; or 'by four resistances in series, each having a bore of 5 sq. mm., provided they were sufficiently widely separated.

\section{Discussion}

The experiments in vivo demonstrate that the cross-sectional area of an effective constriction is unrelated to the normal area of the artery and also that the human carotid artery must be very severely narrowed before there is any change of pressure gradient or of flow. This is to be expected from simple physiological principles; even in the cerebral circulation the major peripheral resistance lies in the smallest vessels, the large vessels contributing very little. A moderate constriction of a large artery, though it may increase large-vessel resistance many times, has disproportionately small effects on the total peripheral resistance, and a very tight constriction is necessary before large-vessel resistance approaches a significant fraction of the total resistance.

Spontaneous atherosclerotic stenosis is much more varied in shape than the standardized constrictions we have studied; their inflow and outflow tracts tend to be curved, and their lumens tend to be irregularly round or oval rather than slit-like, but the general effects of shape and length must still be valid. Allowing for these differences, we can correlate the crosssectional area of a stenosis with its possible effects on blood flow. It is very improbable that a stenosis is significant if its minimal cross-section is more than 5 sq. mm., even if it occupies the whole length of the internal carotid, and this is also true for several stenoses in series ; it is very probable that a stenosis is significant if its minimal cross-section is less than 2 sq. mm., however short it is ; between these two extremes of area stenoses may or may not be significant, depending on their shape and length, and their importance can only be determined by experiment.

These results in one carotid artery are not modified by the presence of additional stenoses in the other three extracranial arteries if none of them constitute a " critical " resistance.

It is difficult to apply these criteria to reports of the incidence of atherosclerosis in cadavers which use the W.H.O. classification in terms of the percentage reduction of the normal lumen, since we find that the bore of a "critical" constriction is unrelated to the normal lumen. The normal lumen of the common carotid artery in our experiments ranged from 17 to
55 sq. mm., with a mean of 30 sq. mm., and the stenosis which we would regard as " possibly significant" with a cross-sectional area of 2-5 sq. mm. corresponds in the W.H.O. classification to an average reduction of the lumen by $84 \%$ to $93 \%$; the internal carotid artery has a lumen somewhat less than the common carotid, but, even allowing for this, many of the stenoses classified as severe by Hutchinson and Yates (1957, 1961) and by Schwartz and Mitchell (1961) almost certainly had negligible effects on resistance.

We suggest that, in future surveys, stenotic lesions in arteries should be classified in terms of their hydraulic resistance measured in a perfusion circuit, using transmural pressures, viscosity, and flow rate corresponding to those present in vivo; where this is impossible the stenosis should be classified in terms of its absolute dimensions rather than in relation to the unstenosed region of the artery.

\section{Summary}

We have determined the dimensions of the constriction which just produces a detectable change of pressure gradient and flow in the internal carotid artery of conscious human subjects.

This " critical" constriction has been duplicated in a perfusion circuit, its hydraulic resistance measured and compared with the resistance of other constrictions of different shapes and lengths.

It is concluded that a stenosis will significantly reduce blood flow if its cross-sectional area is less than 2 sq. $\mathrm{mm}$. If the minimal area is more than 5 sq. $\mathrm{mm}$. blood flow will not be reduced, even by a long stenosis or by several stenoses in series. If the minimal lesion is between 2 and 5 sq. mm., blood flow may be reduced, depending on the shape, on the number of stenoses in series, and, to a less extent, on the length of the stenosis.

We are indebted to the surgeons at Atkinson Morley's Hospital, London, for the opportunity to study cases under their care; to the National Institutes of Health, Bethesda, U.S.A., for the cost of the apparatus; and to the Wellcome Trust for financial support to two of us (D. J.D. and R. D. L.).

\section{REFERENCES}

Brice, J. G., Dowsett, D. J., and Lowe, R. D. (1964). Lancet, 1, 84. Hutchinson, E. C., and Yates, P. O. (1957). Ibid., 1, 2. Schwartz, C. J., and Mitchell, J. R. A. (1961). Brit. med. F., 2, 1057 Yates, P. O., and Hutchinson, E. C. (1961). Spec. Rep. Ser. med. Res. Coun. (Lond.), No. 300.

\title{
Formimino-glutamic Acid Excretion in Anaemia of Pregnancy
}

\author{
MORAG CHISHOLM,* M.B., CH.B.; A. A. SHARP,* M.A., M.D., B.SC., M.C.PATH.
}

When anaemia develops during pregnancy it is usually due to iron deficiency (Fisher and Biggs, 1955), though physiological anaemia due to hydraemia may play a minor part (Lawrence, 1962). Megaloblastic anaemia responding to folic acid can occur in some patients, but the recognition of this condition in pregnancy may be difficult. Unless megaloblasts can be demonstrated in the peripheral blood or buffy coat, a bone-marrow biopsy is necessary before a definite diagnosis can be made. Even with the aid of bone-marrow biopsy

* Haematology Department, Radcliffe Infirmary, Oxford. it may be difficult to be certain that haematopoiesis is abnormal.

This investigation was undertaken to see if the measurement of formimino-glutamic acid (Figlu) in the urine could be used instead of bone-marrow biopsy and could provide a method of determining whether folic-acid deficiency was present in any given patient. This test has been found to be useful in detecting folic-acid deficiency in non-pregnant individuals (Kohn et al., 1961 ; Luhby et al., 1959 ; Knowles et al., 1960), but reports of its value in anaemia of pregnancy have been conflicting (Hibbard, 1962 ; Chanarin et al., 1963). 